\title{
Anomalous Density-of-States Fluctuations in Two-Dimensional Clean Metals
}

\author{
T.R. Kirkpatrick ${ }^{1}$ and D. Belitz ${ }^{2}$ \\ ${ }^{1}$ Institute for Physical Science and Technology and Department of Physics, \\ University of Maryland, College Park, MD 20742 \\ ${ }^{2}$ Department of Physics and Theoretical Science Institute, University of Oregon, Eugene, OR 97403
}

(Dated: September 24, 2018)

\begin{abstract}
It is shown that density-of-states fluctuations, which can be interpreted as the order-parameter susceptibility $\chi_{\mathrm{OP}}$ in a Fermi liquid, are anomalously strong as a result of the existence of Goldstone modes and associated strong fluctuations. In a $2-d$ system with a long-range Coulomb interaction, a suitably defined $\chi_{\mathrm{OP}}$ diverges as $1 / T^{2}$ as a function of temperature in the limit of small wavenumber and frequency. In contrast, standard statistics suggest $\chi_{\mathrm{OP}}=O(T)$, a discrepancy of three powers of $T$. The reasons behind this surprising prediction, as well as ways to observe it, are discussed.
\end{abstract}

PACS numbers: 73.40.Gk, 71.20.-b

The ordered phase in a classical Heisenberg ferromagnet can be understood as a stable fixed point (FP) in a renormalization-group (RG) framework [1, 2]. Analogously, the Fermi-liquid (FL) state in a many-electron system can be understood as an ordered state. This notion was pioneered by Wegner in the context of disordered systems [3]. More recently it was realized that the principle behind it is much more general and powerful, and can be applied to clean FLs as well [4, 5]. An important feature of the resulting effective field theory [5] is that it integrates out all massive degrees of freedom to arrive at an effective soft-mode theory that allows for a RG analysis [6]. As we will show in this Letter, it allows in particular for an analysis of the FL FP, associated corrections to scaling, and the scaling behavior of various observables. Within this framework, the order parameter (OP) is the density of states (DOS) [7], the field conjugate to the $\mathrm{OP}$ is a frequency-dependent chemical potential, and the spontaneously broken continuous symmetry is a rotational symmetry in frequency space that can be understood as the symmetry between retarded and advanced degrees of freedom. These quantities are analogous to the magnetization, the magnetic field, and the spin-rotational symmetry, respectively, in a ferromagnet. The resulting Goldstone modes are the soft particle-hole excitations, i.e., four-fermion correlation functions that mix advanced and retarded degrees of freedom. Their frequency $\omega$ scales linearly with the wave number $k$, and they are responsible, inter alia, for the characteristic $|\omega| / k$ dependence of the Lindhard function 8]. They correspond to the magnons in the ferromagnetic analogy; however, in contrast to magnons they are soft only at zero temperature $(T=0)$.

Let us recall the behavior of the DOS in a FL as a function of the frequency $\omega$ (or the energy distance from the Fermi surface). We will focus on two-dimensional (2- $d$ ) systems, and will consider both a long-ranged Coulomb interaction and a short-ranged interaction. Results for general dimensions $d>1$ will be reported elsewhere [9]. For a Coulomb interaction, it is known that the DOS in $d=2$ is a nonanalytic function of $\omega$, namely,

$$
N(\omega)=2 N_{\mathrm{F}}\left(1+a|\omega| / \epsilon_{\mathrm{F}}\right)+o(\omega)
$$

Here $N_{\mathrm{F}}$ is the DOS per spin at the Fermi level $\epsilon_{\mathrm{F}}$, and $a$ is a coefficient of $O(1) . o(\omega)$ denotes terms that vanish faster than linearly for $\omega \rightarrow 0$. This result has been derived within many-body perturbation theory, which yields a weak-coupling value for $a$, viz., $a=1 / 4 \quad 10 \quad 12]$. As we will show below, the soft-mode theory developed in Ref. 5 establishes the $|\omega|$ as the exact leading frequency dependence, which is something that perturbation theory cannot achieve. Technically, we will show that the frequency correction in Eq. (11) is the leading correction to scaling at the FL FP. For the prefactor, to leading order in a loop expansion we recover the result from perturbation theory, $a=1 / 4$; this will acquire corrections if one goes to higher-loop order. The linear frequency dependence is also consistent with simple scaling considerations, which predict $N(\omega)-N_{\mathrm{F}} \propto|\omega|^{d-1}[5]$, and it constitutes a pseudogap as originally defined by Mott [13]. It is worth noting that this pseudogap is an intrinsic feature of any 2- $d$ Fermi liquid. It has been observed in high-mobility tunnel junctions [14].

Given these results, the behavior of the OP susceptibility is of great interest. This quantity is an important measure of the strength of fluctuations in the ordered phase, which provides a measure of the likelihood that the ordered phase will become unstable as a function of a suitable parameter. In the case of the classical Heisenberg ferromagnet, the OP susceptibility is the magnetic susceptibility $\chi_{\mathrm{m}}=\partial m / \partial h$. As a function of the wave number $k$, the longitudinal component of $\chi_{\mathrm{m}}$ diverges for $k \rightarrow 0$ for all dimensions $d<4: \chi_{\mathrm{m}, \mathrm{L}}(k) \propto 1 / k^{4-d}[15]$. This reflects the strong fluctuations in the ordered phase due to the existence of magnons that couple to the OP. It is then natural to ask whether an analogous susceptibility exists in the FL, and what its behavior at small wave numbers and frequencies is.

To answer this question, we represent the DOS as

$$
N(\omega)=\operatorname{Re} G\left(\boldsymbol{x}=0, i \omega_{n} \rightarrow \omega+i 0\right) / \pi
$$


in terms of the exact Green's function

$$
G\left(\boldsymbol{x}=0, i \omega_{n}\right)=\left\langle\rho\left(\boldsymbol{x}, i \omega_{n}\right)\right\rangle,
$$

with $\rho\left(\boldsymbol{x}, i \omega_{n}\right)=\bar{\psi}_{n}(\boldsymbol{x}) \psi_{n}(\boldsymbol{x})$ in terms of fermion fields $\bar{\psi}_{n}(\boldsymbol{x})$ and $\psi_{n}(\boldsymbol{x})$ that depend on a fermionic Matsubara frequency $\omega_{n}=2 \pi T(n+1 / 2),(n=0, \pm 1, \ldots)$ [16]. We suppress the spin degree of freedom, which is not important for our purposes. The temporal Fourier transform is defined as

$$
\psi_{n}(\boldsymbol{x})=T^{1 / 2} \int_{0}^{1 / T} d \tau e^{i \omega_{n} \tau} \psi(\boldsymbol{x}, \tau),
$$

with $\tau$ the imaginary-time variable. Now we consider the spatial Fourier transform $\rho\left(\boldsymbol{k}, i \omega_{n}\right)=$ $\int d \boldsymbol{x} e^{-i \boldsymbol{k} \cdot \boldsymbol{x}} \rho\left(\boldsymbol{x}, i \omega_{n}\right)$, and the fluctuation $\delta \rho=\rho-\langle\rho\rangle$, and define a correlation function

$$
\chi_{\mathrm{OP}}\left(\boldsymbol{k}, i \omega_{n}\right)=\left\langle\delta \rho\left(\boldsymbol{k}, i \omega_{n}\right) \delta \rho\left(-\boldsymbol{k}, i \omega_{n}\right)\right\rangle .
$$

$\chi_{\mathrm{OP}}$ is the OP susceptibility of interest. It describes both the response of the OP (i.e., the DOS) to its conjugate field, and its spontaneous fluctuations. The former is related to the latter by the fluctuation-dissipation theorem.

We first use simple statistical arguments to determine the behavior of $\chi_{\mathrm{OP}}$ one would expect in the absence of anomalous fluctuations. This will also make a general point that will be important later. Consider $\varphi_{n}(\boldsymbol{x})=$ $\psi_{n}(\boldsymbol{x}) / \sqrt{T}$, and define the "volume" $V_{T} \equiv 1 / T$ in the imaginary-time direction of the space-time of quantum statistical mechanics. Then $\left\langle p_{n}(\boldsymbol{x})\right\rangle \equiv\left\langle\bar{\varphi}_{n}(\boldsymbol{x}) \varphi_{n}(\boldsymbol{x})\right\rangle \propto$ $V_{T}$ is a "time-extensive" quantity that is proportional to $V_{T} \equiv 1 / T$. Now consider the relative fluctuation $\left\langle\left(\delta p_{n}(\boldsymbol{x})\right)^{2}\right\rangle /\left\langle p_{n}(\boldsymbol{x})\right\rangle^{2} \propto V_{T} / V_{T}^{2}=1 / V_{T}=T$. This just says that the relative fluctuation is proportional to $1 / V_{T}$, as one would expect from ordinary statistics. This yields an estimate for the fluctuations of $\rho$ :

$$
\left\langle\left(\delta \rho_{n}\left(\boldsymbol{x}, i \omega_{n}\right)\right)^{2}\right\rangle \propto \frac{\left\langle\left(\delta p_{n}\right)^{2}\right\rangle}{V_{T}^{2}} \propto \frac{\left\langle\left(\delta p_{n}\right)^{2}\right\rangle / V_{T}^{2}}{\left(\left\langle p_{n}\right\rangle / V_{T}\right)^{2}} \propto 1 / V_{T}=T .
$$

These arguments assume that there are no strong fluctuations in the system that invalidate the simple statistics. Independent of this assumption, however, they show that $\left\langle\left(\delta \rho_{n}\left(\boldsymbol{x}, i \omega_{n}\right)\right)^{2}\right\rangle$, and hence $\chi_{\mathrm{OP}}$, must carry a factor of $T=1 / V_{T}$ that is the inverse linear extension of the imaginary-time axis. We thus have

$$
\chi_{\mathrm{OP}}\left(\boldsymbol{k}, i \omega_{n} ; T\right)=T \theta\left(\boldsymbol{k}, i \omega_{n} ; T\right) .
$$

In the absence of anomalous fluctuations, $\theta$ will scale as the zeroth power of the wave number, the frequency, or the temperature; i.e., $\theta \sim 1$.

The last conclusion is not correct in an actual FL. To see this, we first give a phenomenological scaling argument; later we show how to derive scaling from the effective field theory of Ref. 5. We first consider a shortranged interaction, in which case there is only one dynamical scaling exponent $z=1$. This reflects the fact that in the Goldstone modes of the FL, the frequency scales as the wave number, $\omega \sim k$. The DOS correction $\delta N=N-2 N_{\mathrm{F}}$, which is the scaling part of the OP density, is dimensionally an inverse volume times an inverse energy. In $d=2$, and with $z=1$, it thus is expected to scale as an inverse length. The scaling assumption therefore is that $\delta N$ obeys the homogeneity law

$$
\delta N(T)=b^{-1} \delta N(T b) .
$$

This immediately yields $\delta N(T) \propto T$, in agreement with Eq. (11). To obtain the behavior of the OP susceptibility, we remember that the field $h$ conjugate to the OP is a generalized chemical potential, which has a scale dimension $[h]=z$. Adding $h$ as an argument of $\delta N$ in Eq. (8), differentiating with respect to $h$, and adding the wave number argument yields for $\chi_{\mathrm{OP}}=\partial \delta N / \partial h$

$$
\chi_{\mathrm{OP}}(T, k)=\chi_{\mathrm{OP}}(T b, k b)=f_{\chi}(T / k)
$$

with $f_{\chi}$ a scaling function. If we combine this with the conclusion from Eq. (7) that $\chi_{\mathrm{OP}}$ must be proportional to $T$ for fundamental quantum statistical reasons, we find

$$
\chi_{\mathrm{OP}}(T, k) \propto \begin{cases}T / k & \text { for } T \rightarrow 0 \text { at fixed } k \\ T / T=O(1) & \text { for } k \rightarrow 0 \text { at fixed } T .\end{cases}
$$

This result is not consistent with the naive statistical arguments given above: If the DOS were normally distributed, we would have $\chi_{\mathrm{OP}} \propto 1 / V_{T}$ and $\theta \sim 1$. Instead, we see that the quantity $\theta$ in Eq. (7) scales as $\theta \sim 1 / k \sim 1 / T$, and $\chi_{\mathrm{OP}} \sim 1$. This divergence of the relative DOS fluctuations reflects the strong fluctuations in the system that are a consequence of the existence of the Goldstone modes. As we will see below, a long-ranged Coulomb interaction further amplifies these effects.

Before we turn to the case of a Coulomb interaction, let us show how these results can be derived without invoking a scaling assumption, by performing a RG analysis of the effective field theory of Ref. 5. We note that even though the FL FP is not a critical FP, it nevertheless displays scale invariance due to the existence of Goldstone modes. Therefore, very useful results for the entire FL phase can be obtained from very simple RG arguments.

The theory is formulated in term of a soft matrix field $q_{n m}(\boldsymbol{k})$ [17, 18], which encodes the soft components of bilinear fermion fields $\bar{\psi}_{n} \psi_{m}$, viz., those products with $n m<0$. Their softness is guaranteed by a Ward identity. The effective action $\mathcal{A}$ takes the form of an expansion in powers of $q$, see Eqs. (4.51) in Ref. 5 . In a symbolic notation that shows only quantities that carry a scale dimension, viz., the fields $q_{n m}(\boldsymbol{k}) \equiv q$, and factors of volume $V$, wave number $k$, and frequency $\omega$ (which we do not need to distinguish from factors of temperature 
for our purposes), it takes the form

$$
\begin{gathered}
\mathcal{A}=\frac{1}{V} \sum_{k, \omega}[k+\omega+\gamma \omega] q^{2}+\frac{c_{3}}{V^{2}} \sum_{\{k, \omega\}}\left[\omega+O\left(\omega^{3}\right)\right] q^{3} \\
+\frac{c_{4}}{V^{3}} \sum_{\{k, \omega\}}\left[k+\omega+\omega^{2} / k+O\left(\omega^{3}\right)\right] q^{4}+O\left(q^{5}\right) .
\end{gathered}
$$

Here the sums are over the appropriate sets of wave vectors and frequencies, and the powers of $k$ and $\omega$ in each term follow from the behavior of the convolutions of Green's functions that make up the vertices of the theory in the limit of long wavelengths and small frequencies, see Ref. 5. As mentioned above, $\omega$ can stand for either frequency or temperature, and $\gamma$ represents the interaction amplitude. $c_{3}$ and $c_{4}$ are schematic coupling constants; $c_{3} \propto \gamma$. We now determine the FP action that describes the FL. We use Ma's method of choosing scale dimensions for all relevant quantities and then showing self-consistently that these choices lead to a stable FP [2]. We assign a scale dimension $[k]=1$ to wave numbers, and $[\omega]=1$ to frequencies (i.e., we choose a dynamical exponent $z=1$ ). The latter choice reflects the linear dispersion relation of the soft modes, see the first term in Eq. (11), which in a FL we do not expect to be changed by renormalization. We further do not expect the power of wave number (or frequency) in the Gaussian vertex to be renormalized, and therefore assign a scale dimension $[q(\boldsymbol{k})]=-(d+1) / 2$ and $[q(\boldsymbol{x})]=-(d-1) / 2$ to the field as a function of $\boldsymbol{k}$ and $\boldsymbol{x}$, respectively (i.e., we choose the exponent $\eta$ to be zero.) With these choices, the $q^{2}$ term in Eq. (11) is dimensionless; in particular, $[\gamma]=0$. For the cubic term we have $\left[c_{3}\right]=-(d-1) / 2$, for the quartic one, $\left[c_{4}\right]=-(d-1)$, etc. Each additional power of $q$ reduces the scale dimension of the corresponding coupling constant by $-(d-1) / 2$. The FP action is thus given by the Gaussian term alone, and all terms of higher order in $q$ are irrelevant with respect to the FL $\mathrm{FP}$ in all dimensions $d>1$. It follows by standard arguments [1] that this remains true order by order in a loop expansion. All coefficients will in general acquire finite renormalizations, but the structure of the theory will not change. An important ingredient in this chain of arguments is the Ward identity proven in Ref. 5, which identifies $q$ as a soft mode. This assures that the vertices in Eq. (11) will remain soft under renormalization.

We now use the above conclusions to determine the observables we are interested in. Let us first consider the DOS, Eqs. (2, 3). It is given as an expectation value of $\bar{\psi}_{n} \psi_{n}$, which is a massive mode. However, it couples to the soft modes and hence can be expressed as a series of $q$-correlation functions [19]. Schematically,

$$
N \sim 1+\left\langle q^{2}\right\rangle+\left\langle q^{4}\right\rangle+\ldots
$$

The RG arguments given above guarantee that the leading contribution to the DOS correction is given by the term quadratic in $q$. For the scale dimension of the leading scaling part of $\delta N$ this implies $[\delta N]=2[q(\boldsymbol{x})]=d-1$. For $d=2$ this yields Eq. (8). By an analogous argument we find $\left[\chi_{\mathrm{OP}}\right]=d-1-z=d-2$, which for $d=2$ yields Eq. (9). We thus have derived scaling from the field theory via a $R G$ treatment.

In order to determine the correct scaling behavior in the case of a Coulomb interaction, an explicit calculation is needed in addition to general arguments because of the presence of a dangerous irrelevant variable (DIV). This is analogous to the case of a classical ferromagnet in $d>4$, where one needs to explicitly calculate the equation of state to understand why hyperscaling breaks down [2]. To study this case, we replace the constant interaction amplitude $\gamma$ by the dynamically screened Coulomb potential. In $d=2$, the latter has the schematic structure

$$
U(k, i \omega)=\frac{1}{k+\kappa-\frac{\kappa \omega / v_{\mathrm{F}} k}{\sqrt{1+\omega^{2} /\left(v_{\mathrm{F}} k\right)^{2}}}} \approx \frac{1}{u k+\kappa\left(v_{\mathrm{F}} k\right)^{2} / \omega^{2}} .
$$

Here $\kappa$ is the screening wave number, and $v_{\mathrm{F}}$ is the Fermi velocity. The second expression is valid in the limit $v_{\mathrm{F}} k \ll \omega$, and we have introduced a coupling constant $u$ whose bare value is equal to 1 . In the denominator we recognize the plasmon excitation, with its characteristic $\omega \sim k^{1 / 2}$ scaling. We also see the well-known fact that screening breaks down at nonzero frequencies. Counting powers again, we see that $u$ is irrelevant with scale dimension $[u]=-1$. However, it turns out that $u$ is a DIV in $d=2$ with respect to $\chi_{\mathrm{OP}}$ (but not to the DOS).

To demonstrate this we first calculate the $\left\langle q^{2}\right\rangle$ term in Eq. (12), which is the leading contribution to $\delta N$. Using the formalism of Ref. 5 we find that it takes the form of a frequency-momentum integral over a vertex function $V(k, i \omega)$ times $U(k, i \omega)$. The former has the structure $V(k, i \omega)=v\left(\omega / v_{\mathrm{F}} k\right) / k^{2}$, with $v(x \rightarrow \infty) \propto 1 / x^{2}$. The integral that represents the DOS correction then has the structure [20]

$$
\delta N \sim \int_{0}^{\Lambda} d k k \int_{T}^{v_{\mathrm{F}} \Lambda} d \omega \frac{1}{k^{2}} v\left(\omega / v_{\mathrm{F}} k\right) U(k, i \omega)
$$

where $\Lambda$ is an ultraviolet momentum cutoff. From Eq. (14) we see by power counting that $N(T) \propto$ const. $-T$, in agreement with Eqs. (8), (11). This is true for both the long-range and the short-range cases (the latter is recovered by replacing $U$ with a constant); the singular nature of the screened Coulomb interaction at $\omega \neq 0$ does not suffice to change the behavior in $d=2$ 21].

Now consider the OP susceptibility for $d=2$. It follows from Eq. (5) that $\chi_{\mathrm{OP}}$ is given by an integral analogous to the one in Eq. (14) with the integrand squared and an extra factor of $T$. An external wave number effectively serves as a lower cutoff on the wave-number integral, and 
we have schematically

$$
\chi_{\mathrm{OP}}(T, k) \sim T \int_{k}^{\Lambda} d p p \int_{T}^{v_{\mathrm{F}} \Lambda} d \omega\left(\frac{1}{p^{2}} v\left(\omega / v_{\mathrm{F}} p\right) U(p, i \omega)\right)^{2} .
$$

The prefactor of $T$ is just the trivial "volume" factor $1 / V_{T}$ discussed in the context of Eqs. (6, 7). In the short-range case, it is easy to see that Eq. (15) yields Eq. (10) in the respective limits. In the long-range case, using Eq. (13) in Eq. (15) it is easy to see that the integral diverges as $1 / u^{3 / 2}$ for $u \rightarrow 0$. Restoring the prefactors, we find

$$
\chi_{\mathrm{OP}}(k)=\frac{\kappa^{2}}{90 \pi^{2}} \frac{T}{T^{3}} \ln \left(T^{2} / v_{\mathrm{F}}^{2} \kappa k\right)
$$

This result is valid for $T^{2} / v_{\mathrm{F}} k \gg v_{\mathrm{F}} \kappa$, and to logarithmic accuracy. In the opposite limit, we find [22]

$$
\chi_{\mathrm{OP}}(k) \propto \frac{\kappa^{1 / 2}}{v_{\mathrm{F}}^{3}} \frac{T}{k^{3 / 2}},
$$

Finally, for $T \rightarrow 0$ and nonzero external frequency $\omega$ one finds, to logarithmic accuracy for $\omega^{2} \gg v_{\mathrm{F}}^{2} \kappa k$,

$$
\chi_{\mathrm{OP}}(k, i \omega)=\frac{8}{3 \pi^{2}} \frac{\kappa^{2} T}{\omega^{3}} \ln \left(\omega^{2} / v_{\mathrm{F}}^{2} \kappa k\right)
$$

We see that the OP susceptibility, normalized to account for a trivial factor of $T$, Eq. (7), diverges in the limit of vanishing wave number $k$ as $1 / k^{3 / 2}$, or as $1 / T^{3}$ or $1 / \omega^{3}$ in the limits of vanishing temperature or frequency, respectively. This is a very strong effect; uncorrelated statistics would lead to a constant for the same quantity. Comparing Eqs. (16b) and (16a, 16c) we see that $T$ or $\omega$ scale as $k^{1 / 2}$, which reflects the integral being dominated by the plasmon time scale. Although the latter is subleading by power counting, it dominates the scaling due to the DIV $u$. Notice that $\chi_{\mathrm{OP}}(k \rightarrow 0)$ diverges even at a $T>0$ since the plasmon, as a density fluctuation governed by a conservation law, remains soft even at $T>0$.

Also of interest is the homogeneous susceptibility in a finite system of linear dimension $L$. In that case, $k$ in Eqs. (16) gets replaced by $1 / L$ [9].

We finally add some comments about the experimental relevance of the above results. The spectrum of the local Green's function, Eq. (3), is what is often referred to as the local density of states (LDOS) 23, 24]. The LDOS gives the dominant contribution to the tunneling current in a scanning tunneling experiment 25]; its spatial average, which is the DOS, is measured in a classic tunnel junction. Our OP susceptibility, Eq. (5), describes the spatially averaged fluctuations of the LDOS. A two-tip tunneling experiment [23, 26] should be able to give information about this quantity.

We are grateful to S. Gregory and H. Manoharan for helpful discussions. This work was supported by the National Science Foundation under Grant Nos. DMR-0929966, DMR-09-01907, and PHY-10-66293. Part of this work was performed at the Aspen Center for Physics.
[1] K. G. Wilson and J. Kogut, Phys. Rep. 12, 75 (1974).

[2] S.-K. Ma, Modern Theory of Critical Phenomena (Benjamin, Reading, MA, 1976).

[3] F. Wegner, Z. Phys. B 35, 207 (1979).

[4] D. Belitz and T. R. Kirkpatrick, Phys. Rev. B 56, 6513 (1997).

[5] T. R. Kirkpatrick and D. Belitz, Phys. Rev. Lett. 108, 086404 (2012); D. Belitz and T. R. Kirkpatrick, Phys. Rev. B 85, 125126 (2012).

[6] The separation of soft and massive modes sets this theory apart from the treatment by R. Shankar, Rev. Mod. Phys. 66, 129 (1994), which kept all degrees of freedom since it had a different purpose. An similar, but technically different, approach has been developed by I.L. Aleiner and K.B. Efetov, Phys. Rev. B 74, 075102 (2006), K.B. Efetov, C. Pépin, and H. Meier, Phys. Rev. B 82, 235120 (2010) and H. Meier, C. Pèpin and K.B. Efetov, Phys. Rev. B 84, 205131 (2011). Also similar in spirit is the ballistic nonlinear sigma model, B.A. Muzykantskii and D.E. Khmelnitskii, JETP Lett. 62, 76 (1995), which describes weakly disordered systems whose physics is different from the effects we discuss.

[7] More precisely, the OP is the single-particle spectral density, whose zeroth wave-number moment is the DOS. For simplicity, we refer to the DOS as the OP.

[8] D. Pines and P. Nozières, The Theory of Quantum Liquids (Addison-Wesley, Redwood City, CA, 1989).

[9] D. Belitz and T.R. Kirkpatrick, to be published.

[10] D. V. Khveshchenko and M. Reizer, Phys. Rev. B 57, R4245 (1990). These authors found $a=1 / 2$. We believe this is due to a simple algebraic mistake, rather than a missing physical contribution as suggested in Ref. 11.

[11] E. G. Mishchenko and A. V. Andreev, Phys. Rev. B 65, 235310 (2002).

[12] This result holds in the long-ranged case. For remarks regarding the short-ranged case, see Ref. 21 .

[13] N. F. Mott, Rev. Mod. Phys. 40, 677 (1968).

[14] See Ref. 27. The interpretation given in this paper was in terms of different physics, in our opinion erroneously so. Also, the pseudogap in the normal phase of the cuprates has been found to not scale with the superconducting properties, Ref. 28. The energy scale in the latter experiment is roughly consistent with Eq. (1), as is the one in Ref. 27. This raises the possibility that the pseudogap in the cuprates can be explained in terms of 2- $d$ Fermi-liquid physics. This notion requires further investigation.

[15] E. Brézin and D. J. Wallace, Phys. Rev. B 7, 1967 (1973).

[16] $\rho\left(\boldsymbol{x}, i \omega_{n}\right)$ can be interpreted as the density of particles at point $\boldsymbol{x}$ and (complex) energy $i \omega_{n}$. Note, however, that $\rho$ is local in frequency space, since it is defined as a product of two fermion fields at the same frequency $\omega_{n}$, whereas the actual particle number density is local in time and involves a convolution in frequency space.

[17] The theory also contains a second field $\phi_{n m}(\boldsymbol{k})$. However, this has the same scale dimension as $q$, and for our purposes we do not need to distinguish between $q$ and $\not$.

[18] We use what in Ref. 5 was called the density formulation of the effective theory, within which $q$ is local in space.

[19] This is in exact analogy to the fact that the massive field $\sigma$ in a classical $O(N)$ nonlinear sigma-model can be expressed as a power series in the soft field $\boldsymbol{\pi}$, see. 
e.g., J. Zinn-Justin, Quantum Field Theory and Critical Phenomena, Oxford University Press (Oxford, 1996).

[20] This, as well as the structure of the vertex, can be seen from Eq. (5.4) in Ref. 5 .

[21] A more careful evaluation shows that in $d=2$ in the short-range case, the $T$ or $|\omega|$ term has a zero prefactor [5], in agreement with earlier results from many-body perturbation theory [11, 29]. In the long-range case, the integral depends logarithmically on the DIV $u$. However, this does not change the scaling behavior of the DOS. In $d<2$ the integral diverges as $u^{-(2-d)(d-1) /(3-d)}$, and $\delta N \propto|\omega|^{(d-1) /(3-d)}[9]$.

[22] The prefactor in Eq. (16b) is difficult to determine since the wave number integral involves a convolution.

[23] Y. S. Chan and E. J. Heller, Phys. Rev. Lett. 78, 2570 (1997)

[24] This definition of the LDOS is more general than the one found in earlier papers, e.g., Ref. 25, as it is in terms of the exact Green's function and thus includes the full many-body effects. We also note that our local Green's function is independent of the position because we consider a homogeneous model; it becomes position dependent if one takes into account the underlying lattice.

[25] J. Tersoff and D. R. Hamann, Phys. Rev. B 31, 805 (1985).

[26] Q. Niu, M. C. Chang, and C. K. Shih, Phys. Rev. B 51, 5502 (1995).

[27] P. Jiang, I. Yang, W. Kang, L. N. Pfeiffer, K. W. Baldwin, and K. W. West, Phys. Rev. Lett. 96, 126804 (2006).

[28] Th. Jacobs et al., arXiv:1202.6370

[29] A. Chubukov, D. Maslov, S. Gangadharaiah, and L. Glazman, Phys. Rev. B 71, 205112 (2005). 